\title{
MISSING MASS METHOD FOR SHORT-LIVED PARTICLE RECONSTRUCTION IN THE CBM AND STAR EXPERIMENTS
}

\author{
Y. Fisyak ${ }^{1}$, V. Ivanov ${ }^{2,3}$, H. Ke ${ }^{1}$, I. Kisel ${ }^{4,5,6,7}$, P. Kisel ${ }^{2,5, a}$, G. Kozlov ${ }^{2,5}$, \\ S. Margetis ${ }^{8}$, A. Tang ${ }^{1}$, I. Vassiliev ${ }^{4}$ \\ ${ }^{1}$ BNL, Brookhaven National Laboratory, Upton, NY, USA \\ ${ }^{2}$ JINR, Joint Institute for Nuclear Research, Dubna, Russian Federation \\ ${ }^{3}$ MEPhI, National Research Nuclear University, Moscow, Russian Federation \\ ${ }^{4}$ GSI, Helmholtz Center for Heavy Ion Research, Darmstadt, Germany \\ ${ }^{5}$ Goethe University, Frankfurt am Main, Germany \\ ${ }^{6}$ FIAS, Frankfurt Institute for Advanced Studies, Frankfurt am Main, Germany \\ ${ }^{7}$ HFHF, Helmholtz Research Academy Hesse, Frankfurt am Main, Germany \\ ${ }^{8}$ KSU, Kent State University, Kent, OH, USA \\ E-mail: ${ }^{a}$ P.Kisel@gsi.de
}

The search for short-lived particles is an important part of physics research in experiments with relativistic heavy ions. To find, select and study the properties of such short-lived particles in real time in the CBM experiment (FAIR/GSI, Germany), we have developed a package of algorithms, KF Particle Finder, which contains a search for more than 150 decay channels. The decays of short-lived charged particles, when one of the daughter particles is neutral and cannot be registered in the detector system, are also of great physics interest. To find and study such decays, we have extended the KF Particle Finder package by implementing the missing mass method, which is based on the conservation of energy and momentum laws.

The method was studied in detail on simulated data of the CBM experiment, showing high efficiency with a large signal-to-background ratio, as well as high significance. As part of the FAIR Phase-0 program, the KF Particle Finder package of algorithms has been adapted for online and offline processing in the STAR experiment (BNL, USA).

Here we describe and discuss in detail the missing mass method for finding and analyzing short-lived particles. The features of the application of the method to both simulated data in the CBM experiment and in the STAR experiment as part of real-time express data processing, as well as the results of the real-time reconstruction of short-lived particle decays in the BES-II environment of the STAR experiment, are given.

Keywords: HPC, parallel programming, heavy-ion collisions, CBM experiment, STAR experiment

Yuri Fisyak, Victor Ivanov, Hongwei Ke, Ivan Kisel, Pavel Kisel, Grigory Kozlov, Spyridon Margetis, Aihong Tang, Iouri Vassiliev 


\section{Introduction}

In modern experiments with heavy ions, such as CBM (FAIR/GSI, Darmstadt, Germany), ALICE (CERN, Geneva, Switzerland) and STAR (BNL, Upton, USA), the main task is a comprehensive study of the QCD phase diagram in the quark-gluon plasma (QGP) region and a possible phase transition from the hadronic to QGP phase.

In this regard, strange particles, which are sensitive probes of the medium formed in heavy-ion collisions, play an important role. Since strange particles have decay modes with at least one neutral daughter particle, their decay products cannot be registered in a tracking detector system. To identify such decays, when one of the daughter particles is neutral, we proposed and implemented the missing mass method.

The missing-mass method was originally developed by us within the FLES (First Level Event Selection) package [1] for the CBM experiment. In the process of developing and investigating the method in CBM, the method has shown its high efficiency on simulated data. However, for the first phase of the CBM experiment, it is important to prove the concept of the developed approach and its current implementation on real data. The FAIR Phase-0 program [2], which includes participation in the STAR experiment at BNL (USA), gives us such an opportunity.

\section{Missing mass method}

The missing mass method is used to reconstruct decays of short-lived charged particles, when one of the daughter particles is neutral and not registered in the detector system. The method is based on the laws of conservation of energy and momentum.

As an illustration of the method, let us consider the well-known decay

$$
\Sigma^{-} \rightarrow \mathrm{n} \pi^{-} .
$$

In this decay, the daughter pion is registered in the tracking system and identified in one of the PID detectors, while the neutron is not detected. Although the sigma particle has a short lifetime $\mathrm{c} \tau_{\Sigma^{-}}=$ $4.4 \mathrm{~cm}$, in fixed-target experiments due to relativistic boost, the average length of its trajectory in the laboratory system can reach $15-20 \mathrm{~cm}$. In this case, the $\Sigma^{-}$particle will produce measurements in several tracking stations and can therefore be reconstructed. Thus, in the given decay, two charged tracks out of total three tracks can be reconstructed.

A specific feature of this type of decays is that the short-lived mother track usually cannot be identified, making it impossible to directly calculate the missing neutron mass. However, the momentum of the mother particle $\Sigma^{-}$, unlike its mass, can be determined from the curvature of the trajectory. Therefore, we can assume and apply the hypothesis about the mass of the mother track, and then look at the resulting neutron mass spectrum.
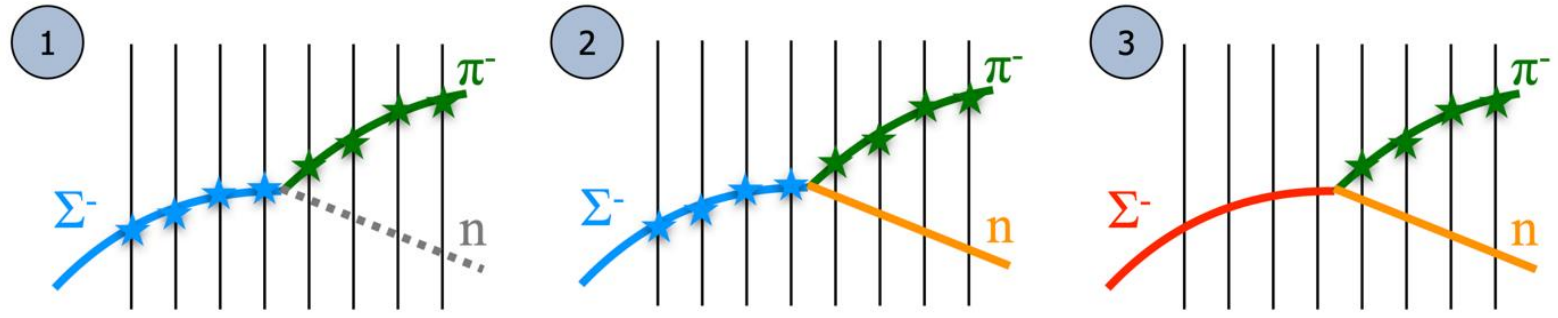

Figure 1. Three stages of the reconstruction of a short-lived charged particle using the missing mass method

Thus, the reconstruction of a short-lived charged particle using the missing mass method is performed in three steps [Fig. 1]: 
1. reconstruction of tracks of the charged mother particle $\left(\Sigma^{-}\right)$and its charged daughter particle $\left(\pi^{-}\right)$in the tracking system;

2. reconstruction of the parameters of the neutral daughter particle (n) by the parameters of the mother particle and the charged daughter;

3. reconstruction of the $\Sigma^{-}$mass spectrum using the charged particle and the obtained neutral daughter.

The method is implemented as an extension of the KF Particle Finder [1, 3] package, which is now actively used to search and reconstruct short-lived particles, both online and offline, in major heavy ion experiments such as CBM (FAIR/GSI), ALICE (CERN) and STAR (BNL). A search for 18 decays with a neutral daughter particle has now been added to the KF Particle Finder package.

Once the particle candidates are found in the search for short-lived particles in the KF Particle Finder, there is competition among them to ensure that the daughter particles participate in only one, the most probable, decay channel. As an example, the decays $\mathrm{K}_{\mathrm{s}}{ }^{0} \rightarrow \pi^{+} \pi^{-}$and $\Lambda \rightarrow \mathrm{p} \pi^{-}$can be given, when in both decays one of the daughter particles is $\pi^{-}$.

Within the KF Particle Finder package, two approaches have been implemented to perform competition between particle candidates with possible common daughter particles: deterministic approach and artificial neural network approach. In the deterministic approach, the reconstruction quality of such competing particle candidates is compared, and on the basis of certain criteria, such as $\chi^{2}$-value and closeness to the table mass value, the best particle is chosen. In the artificial neural network approach, similar to its other applications $[4,5]$ to optimize and select data, the network is first trained on the simulated data and then selects the best particles on its own. Both approaches show almost identical results [6], so the choice of one for data processing is purely practical.

The missing mass method has been repeatedly and successfully tested within the KF Particle Finder package on simulated data of the CBM experiment, demonstrating its value to the physics program of the experiment [7].
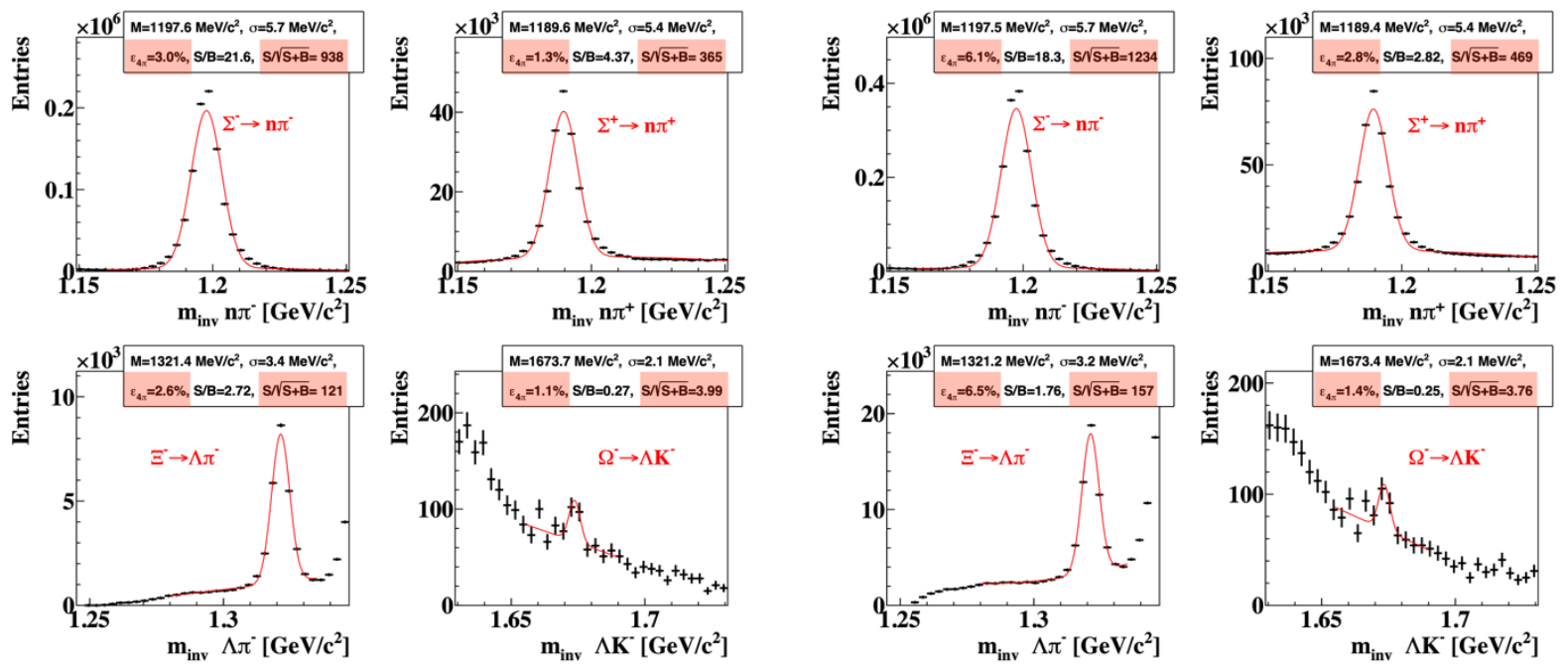

Figure 2. Performance of the missing mass method in the CBM experiment on $5 \mathrm{M}$ central AuAu UrQMD events at $10 \mathrm{AGeV}$ (left: standard version, right: improved version)

After the intensive investigation of the method, we have made numerous improvements to its standard realization. Thus, in the modified realization of the method, the decay point of a charged particle, or its secondary vertex, is in two steps at once at the initial stage and does not require any further correction and recalculation, which is mathematically more accurate. In addition, we have significantly improved the mathematics of the implementation of the method: a more accurate and faster mathematical apparatus has been used; all basic calculations are performed with $6 \times 6$ matrices 
instead of the former $7 \times 7$; the energy has been removed from the state vector and the covariance matrix; the energy and mass calculations are done separately in the final step; the parameters of all decaying particles are estimated in one iteration of the Kalman filter instead of the former two. This has allowed one to avoid the very resource-intensive method of the mass constraint, which had to balance the parameters of the particles in some parts of the calculations.

The modification of the method resulted in a 2-fold increase in efficiency and a 25-30\% increase in significance [Fig. 2].

As part of the FAIR Phase-0 program [2], the missing mass method has been successfully tested on pion and kaon decay channels offline on real STAR data from 2016 and 2020 data sets.
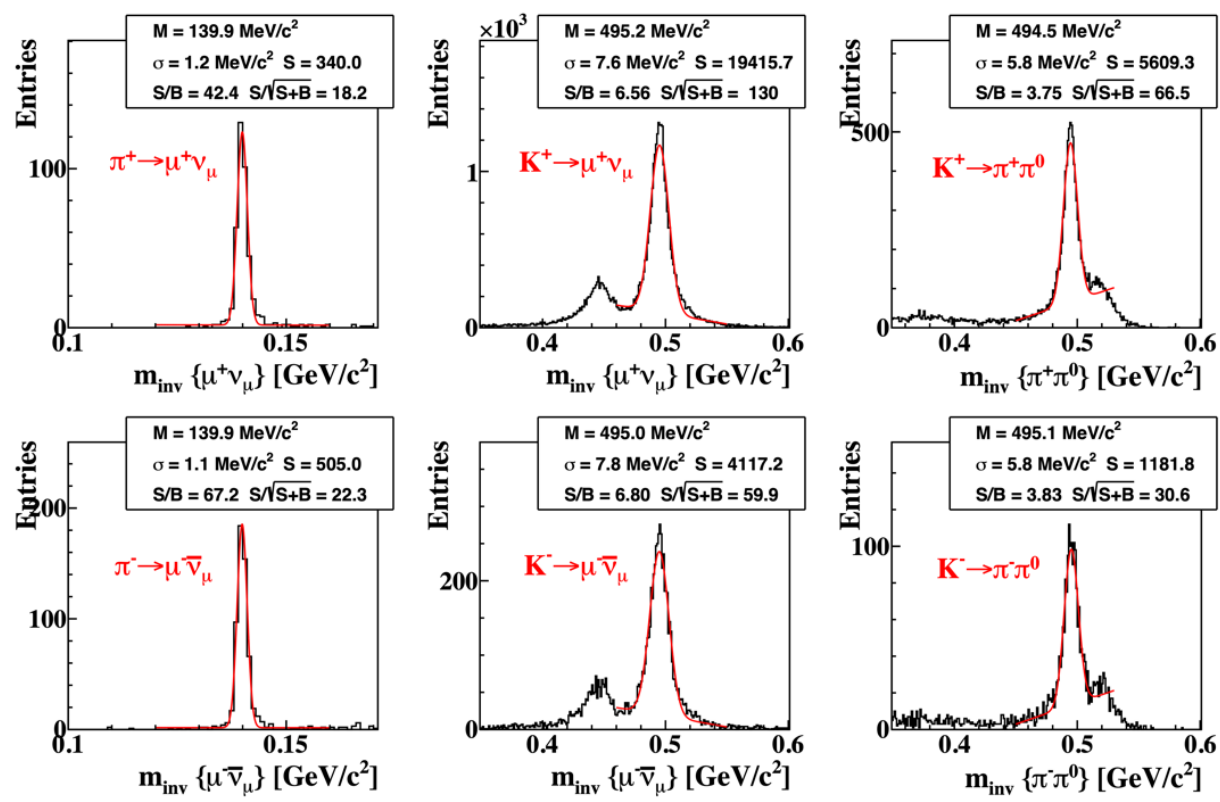

Figure 3. Results of the standard version of the missing mass method on 2M AuAu events of 2020 year data at $(5.75+7.3+9.8+26.5) \mathrm{AGeV}$
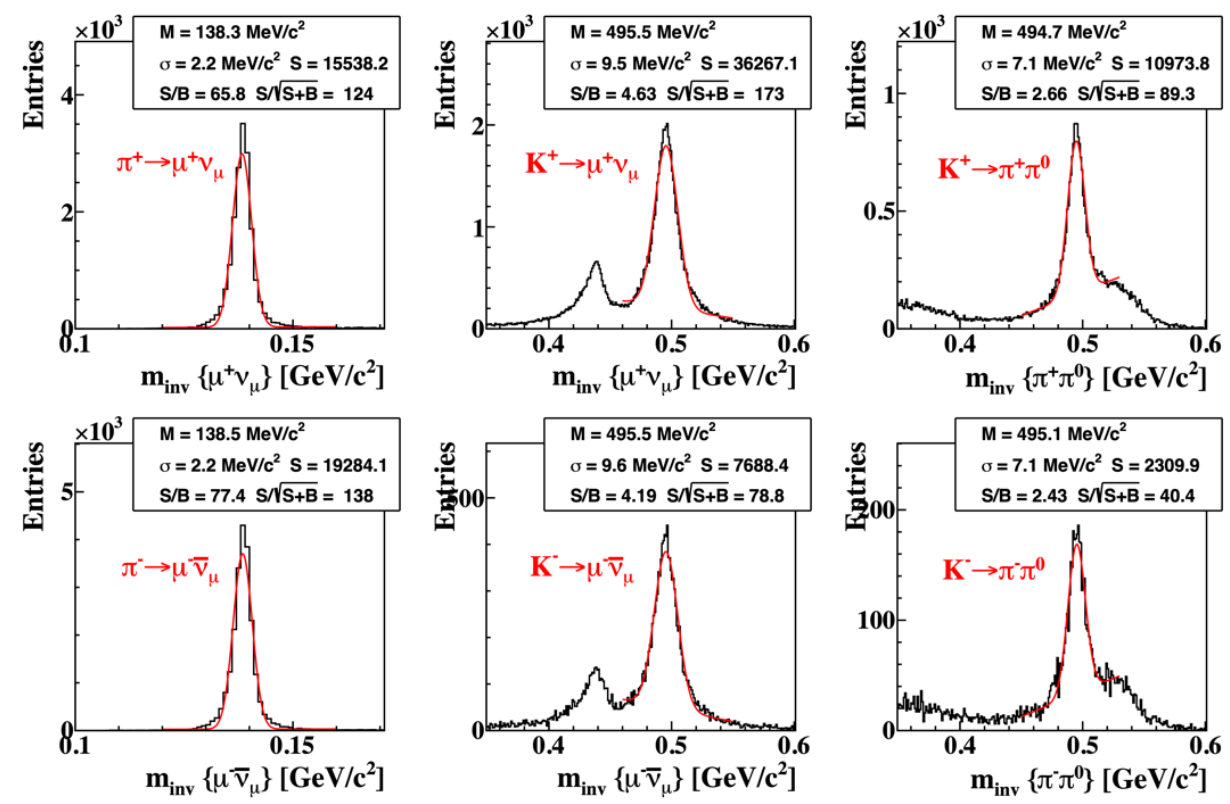

Figure 4. Results of the improved version of the missing mass method on $2 \mathrm{M}$ AuAu events of 2020 year data at $(5.75+7.3+9.8+26.5) \mathrm{AGeV}$ 
Here we present the results of applying the missing mass method to search for pion $\left(\pi^{ \pm} \rightarrow \mu^{ \pm} v\right)$ and kaon $\left(\mathrm{K}^{ \pm} \rightarrow \mu^{ \pm} v\right.$ and $\left.\mathrm{K}^{ \pm} \rightarrow \pi^{ \pm} \pi^{0}\right)$ decays to real data $2 \mathrm{M}$ AuAu events of STAR at $(5.75+7.3+9.8+26.5)$ AGeV collected in 2020 within the Beam Energy Scan program BES-II and reconstructed online running the express data production and analysis chain on the STAR HLT farm.

The standard version of the missing mass method provides the online reconstruction of shortlived particles of 2020 year data with a high signal-to-background ratio and significance [Fig. 3].

In the improved version, in the $\pi^{ \pm} \rightarrow \mu^{ \pm} v$ channels, the signal increased by a factor of 40 , and the significance increased by a factor of 7 . In the $\mathrm{K}^{ \pm} \rightarrow \mu^{ \pm} \nu$ and $\mathrm{K}^{ \pm} \rightarrow \pi^{ \pm} \pi^{0}$ channels, the signal increased by a factor of 2 , and the significance increased by a factor of 1.5 [Fig. 4].

\section{Conclusion}

The missing mass method enables a significant expansion of the physics programs of highenergy and heavy-ion physics experiments. Within the FAIR Phase-0 program, the KF Particle Finder package, initially developed for the CBM experiment, has been adapted to the STAR experiment and applied to real data of BES-II. The missing mass method provides the online reconstruction of real data with a high signal-to-background ratio and significance.

\section{Acknowledgement}

The work is supported by the German Federal Ministry of Education and Research BMBF and the Helmholtz Research Academy Hesse HFHF.

\section{References}

[1] I. Kisel, I. Kulakov, and M. Zyzak, "Standalone First Level Event Selection Package for the CBM Experiment", IEEE Trans. on Nucl. Science, 60, 5, (2013) 3703.

[2] I. Kisel (for CBM and STAR Collaborations), "Real-Time Event Reconstruction and Analysis in CBM and STAR Experiments", Journal of Physics: Conference Series 1602 (2020) 012006.

[3] M. Zyzak, "Online Selection of Short-Lived Particles on Many-Core Computer Architectures in the CBM Experiment at FAIR", Dissertation thesis, Goethe university, Frankfurt am Main (2016).

[4] Artificial neural networks for optimisation in online marketing, https://ann2thrive.com/

[5] F. Sergeev, E. Bratkovskaya, I. Kisel and I. Vassiliev, "Deep learning for quark gluon plasma detection in the CBM experiment", International Journal of Modern Physics A, Vol. 35, No. 33 (2020) 2043002.

[6] A. Banerjee, I. Kisel and M. Zyzak, "Artificial neural network for identification of short-lived particles in the CBM experiment", International Journal of Modern Physics A, Vol. 35, No. 33 (2020) 2043003.

[7] V. Akishina, I. Kisel, P. Kisel, P. Senger, I. Vassiliev, and M. Zyzak, "Reconstruction of Particles Produced at Different Stages of Heavy Ion Collision in the CBM Experiment at FAIR", Poster, Quark Matter 2017, 5-11 February 2017, Chicago, USA. 